\title{
A Multistage System for Automatic Detection of Epileptic Spikes
}

\author{
Nguyen Thi Anh-Dao ${ }^{1,2}$, Nguyen Linh-Trung ${ }^{2}$, Ly Van Nguyen ${ }^{2}$, Tan Tran-Duc ${ }^{2}$, \\ Nguyen The Hoang Anh ${ }^{3}$, Boualem Boashash ${ }^{4}$ \\ ${ }^{1}$ Electronics and Telecommunications, University of Technology and Logistics, Bac Ninh, Viet Nam \\ 2 AVITECH, VNU University of Engineering and Technology, Hanoi, Vietnam \\ ${ }^{3}$ Institute of Information Technology, Vietnam Academy of Science and Technology, Hanoi, Vietnam \\ ${ }^{4}$ UQCCR, Centre for Clinical Research, The University of Queensland, Brisbane, Australia
}

Correspondence: Nguyen Linh-Trung, linhtrung@vnu.edu.vn

Communication: received 12 June 2017, revised 22 January 2018, revised 25 January 2018, accepted 25 January 2018

Online publication: 14 March 2018, Digital Object Identifier: 10.21553/rev-jec.166

The associate editor coordinating the review of this article and recommending it for publication was Dr. Vo Trung Dung.

\begin{abstract}
A multistage automatic detection system for epileptic spikes is introduced as an assistant tool for epileptic analysis and diagnosis based on electroencephalogram (EEG). The system consists of four stages: preprocessing, feature extraction, classifier and expert system. Multiple state-of-the-art signal processing and machine learning techniques including wavelet transform, spectral filtering, artificial neural network are utilized in order to improve the ability of the overall system, stage by stage. Compared to other works, our contributions are three-fold: peaks in the EEG recording are categorized into two groups of non-epileptic spikes and possible epileptic spikes by a committee of three perceptrons; appropriate mother wavelet and wavelet scales are selected for the best system performance; and, based on the neurological fact that an epileptic spike is usually followed by a slow wave, a simple expert system is presented to eliminate pseudo-spikes which are closely analogous to true epileptic spikes. Experimental results show that the proposed system is capable of detecting epileptic spikes efficiently.
\end{abstract}

Keywords- Epilepsy, electroencephalogram (EEG), spike, time-frequency, continuous wavelet transform, artificial neural network, expert system, neurology.

\section{INTRODUCTION}

Epilepsy is a set of chronic neurological disorders, which can be characterized by seizures and epileptiforms. Epileptic seizures result from abnormal, excessive or hyper synchronous neuronal activity in the brain. Epileptiforms are waveforms related to epilepsy, such as spikes, sharp waves and spike-wave complexes and occur before or after a seizure [1]. Scalp electroencephalogram (EEG), which is the recording of electrical activity of the brain, measures voltage fluctuations resulting from ionic current flows within the neurons of the brain by using electrodes placed on the scalp.

Among different tools for epilepsy analysis, scalp EEG remains the most accessible method. Despite limited spatial resolution, EEG continues to be a valuable tool for research and diagnosis, especially when millisecond-range temporal resolution is required. Unlike 24-hour monitoring where one aims to record the occurrence of seizures of a patient, in clinical recording often only epileptiforms are observed. This paper considers the analysis of epileptic spikes in clinical recording in Vietnam.

In the procedure of epilepsy diagnosis, automatic spike detection is important because it can provide much information, such as spike density and patient syndrome. Much effort has been spent on spike detection over the last 40 years. The reader is referred to excellent reviews on the topic that can be consulted in [2-4].

While manual spike detection via visual identification by neurologists is very time consuming, state-ofthe-art automatic spike detection remains difficult for a number of reasons. First, neurologist-based definitions of a spike are not simplistic [2]. Two human neurologists often do not mark the same events as spikes leading to a large ratio of candidate spike events to actual spike events. Second, spike morphology and background vary widely between patients, and well defined training sets are time consuming and expensive to develop. In Vietnam, the application of EEG recording in epilepsy diagnosis is still at a rudimentary stage due to (i) scarcity of professional neurologists who can provide high-quality analysis based on electrical neural information, (ii) shorter period (10 minutes) of clinical recording compared to standard conventional period (20 minutes) and (iii) abundance of artefacts caused by atypical recording environment. The last two reasons often make the job of Vietnamese neurologists become harder than their international colleagues, leading to high rates of false visual identification of epileptiforms. Thus, it is important for Vietnamese biomedical signal processing community to develop a system capable of identifying epileptiforms automatically, in order to 
assist neurologists to improve the quality of epilepsy analysis and diagnosis.

Over the past decades, various methods have been proposed to solve the problem of automatic epilepsy spike detection. Some methods compared the measurements of electrographic parameters of EEG waveforms with representative thresholds of typical true spike (e.g., [5, 6]). Some others (e.g, [7]) proposed some filtering techniques for spike detection. In another approach, the authors in [8] developed a different system for spike detection, which is sensitive to the different states of EEG such as active wakefulness, quiet wakefulness, desynchronized EEG, phasic EEG, and slow EEG.

In several recent reviews, automatic detection methods are categorized into different groups based on neuro-physiological [1] or engineering [4] characteristics of the methods. However, none of the existing categorizations provides an adequate and exact overview for readers on the entire set of spike detection systems.

In this work, automatic detection systems for epileptic spikes are categorized based on their structure into two groups: simplex and multi-stage. On the one hand, systems in the simplex group are the ones with a simple structure, normally consisting of one or two engineering techniques. On the other hand, those in the multi-stage group are built up by combining multiple signal processing and machine learning techniques in order to take advantages of each specific technique over capturing and extracting suited features and over processing and classifying information. Usually, the multi-stage systems outperform the simplex systems in identifying epileptic spikes.

In the simplex group, one of the very first works is the application of an autoregressive model for spike detection. The criteria and the decision making process that the neurologist used in identifying spikes were imitated in [9] for the same task based on the information provided by decomposing EEG signals into elementary waves. In another work [10], EEG signals are analyzed by Independent Component Analysis, and then, components resembling epileptic activities are selected and interpreted by neurologists. Morphological Filters are employed in [11] for the task of epileptic spike detection using geometric characteristics. Some machine learning techniques such as Artificial Neural Networks (ANN) [12, 13], K-means clustering [14] and Support Vector Machines [15] have been used as effective tools for spike classification and detection based on two main approaches, whether using raw EEG or features extracted from raw EEG for model training and testing.

In the multi-stage group, three existing systems were presented by Liu et al. in [16], Hassanpour and Boashash in [17], and Acır and Güzeliş in [18], and, comprising of multiple stages of different signal processing and machine learning models and proved to be effective.

The system in [16] is for 24-hour monitoring and combines multiple signal-processing methods in a multistage scheme that integrates adaptive filtering, wavelet transform, an ANN and an expert system. Inputs of the ANN in the first stage of the system are features extracted after the wavelet transformation of the raw EEG. By doing that, nonstationary components including pseudo-spike artifacts like electromyogram (EMG) artifacts are identified and reduced.

In [17], a two-stage spike detection technique is introduced based on time-frequency distributions to account for the nonstationarity exhibited in EEG signals. In the first stage, noise is reduced in the timefrequency domain based on a singular value decomposition based method, resulting in an enhanced timefrequency distribution of the signal. In the second stage, two frequency slices of this enhanced distribution are extracted and then become the input of the smoothed nonlinear energy operator (SNEO) for spike detection. The system has a high computational complexity because of calculations in the time-frequency domain for the entire data. This is not however a problem if one uses parallel processing efficiently.

Meanwhile, the system in [18] is for clinical monitoring and uses a three-stage procedure for the automatic detection of epileptiform events in a multichannel EEG signal. In the first stage, three discrete perceptrons are fed by six spike features that are used for classifying EEG peaks into three subgroups: (i) definite epileptiform transients (ETs), (ii) definite non-ETs, and (iii) possible ETs and possible non-ETs. Peaks in the third group are further processed. However, time-frequency methods like wavelet transformation or quadratic timefrequency distributions are not utilized, leading to its weak capability in identifying pseudo-spikes which are nonstationary. Nonetheless, multistage systems often provide better performance then simplex systems.

Taking advantage of multi-stage systems, we propose in this paper a multistage system for automatic spike detection that can offer good performance. Our contributions are three-fold. First, EEG peaks are categorized into two groups of (i) non-epileptic spikes and (ii) possible epileptic spikes, by a committee of three perceptrons; each committee is fed by six different features characterizing an epileptic spike. Spikes resulting from EMG-like artefacts, which are continuous within $40 \mathrm{~ms}$ (i.e., 10 data samples for the sampling frequency of $256 \mathrm{~Hz}$ ), are also eliminated by the perceptrons. Second, wavelet transformation is utilized with a Mexican mother wavelet and its corresponding scales from 4 to 8 for the best efficiency. Third, closely located pseudospikes are removed, thanks to the neurological fact that an epileptic spike is often followed by slow waves rather than by another epileptic spike [19]. Experimental results are obtained from analyzing the clinical EEG recordings of 17 epileptic patients.

The paper is organized as follows. Section 2 describes the proposed automatic epileptic seizure detection system. Section 3 gives detailed information on the proposed system and related theoretical foundations. Experimental results and their analysis are presented in Section 4. Section 5 discusses the results and Section 6 concludes the paper with some remarks for future works. 


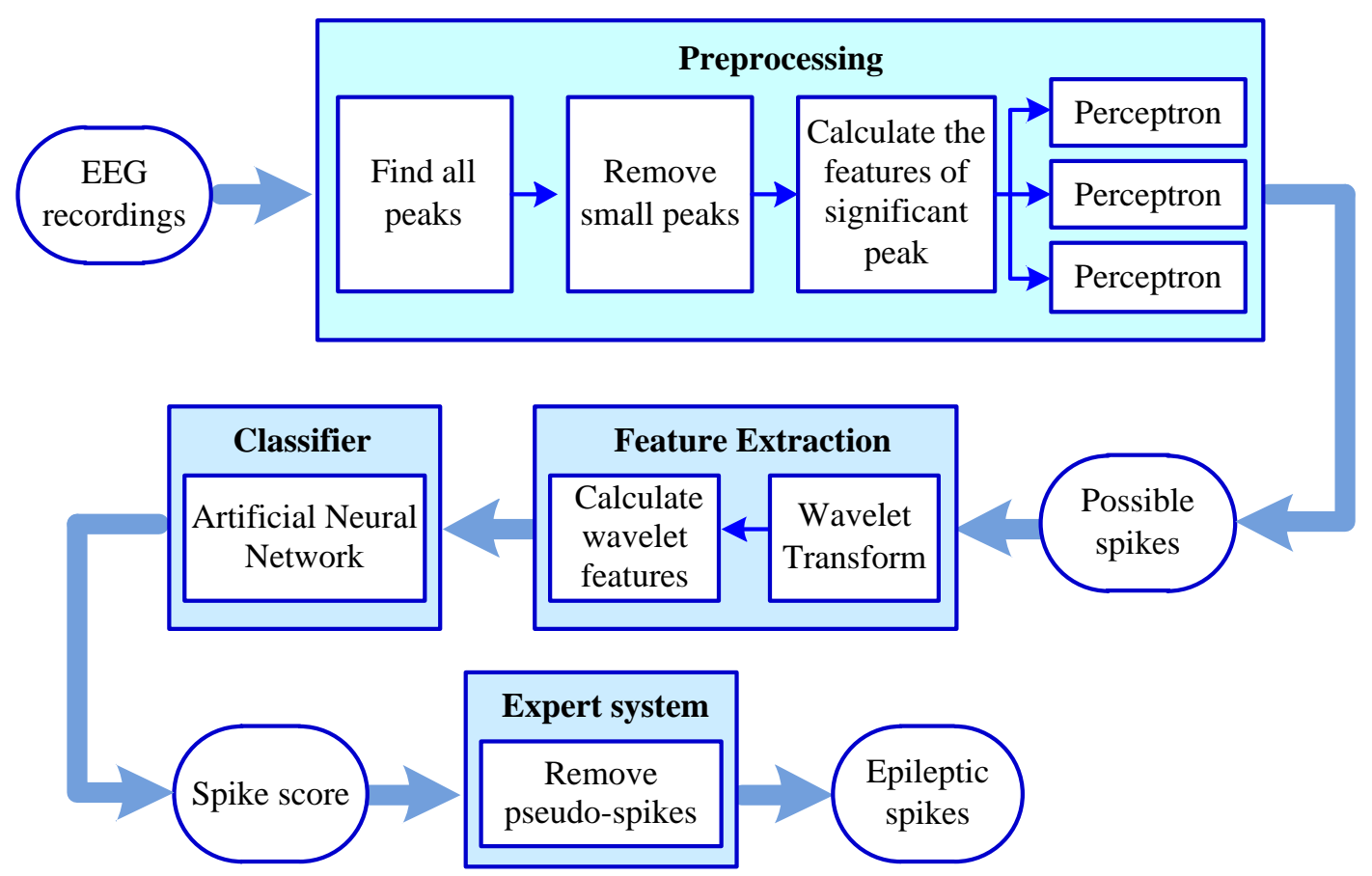

Figure 1. Block diagram of the proposed multi-stage automatic spike detection system, with 4 stages: preprocessing, feature extraction, classifier and expert system.

\section{Proposed System Model}

Figure 1 shows the block diagram of the proposed spike detection system. It consists of 4 stages: Preprocessing, Feature extraction, Classifier and Expert system. The tasks in each stage are briefly given as follows.

In the preprocessing stage, all peaks in an EEG signal are first automatically detected. Then, negligible peaks are identified and removed. Next, for each significant peak, six spike features depicting the amplitudes, durations and slopes associated with the morphology of an epileptic spike are calculated. These features are then fed into three different perceptrons which then separate the significant peaks into two groups of (i) non-epileptic spikes and (ii) possible epileptic spikes.

Next, in the feature extraction stage, the possible epileptic spikes are analyzed by continuous wavelet transform. The proposed system then calculates seven wavelet features of each wavelet scale, which resembles an epileptic spike. Then, in the classifier stage, the extracted features are fed into a trained ANN yielding a spike score at its output. The spike score values are in the range $[0,1]$. If a spike score value is closer to 1 or 0 , then the corresponding spike is likely to be an epileptic spike or non-epileptic spike, respectively.

Finally, in the expert system stage, to ensure that the spike detected by the ANN is a true epileptic spike, we apply a simple rule in order to eliminate the pseudospikes which are located near an epileptic spike.

\section{Methods}

\subsection{Preprocessing}

Epileptic spikes last for a short duration, typically between 20 to $70 \mathrm{~ms}$, and are characterized by a steep

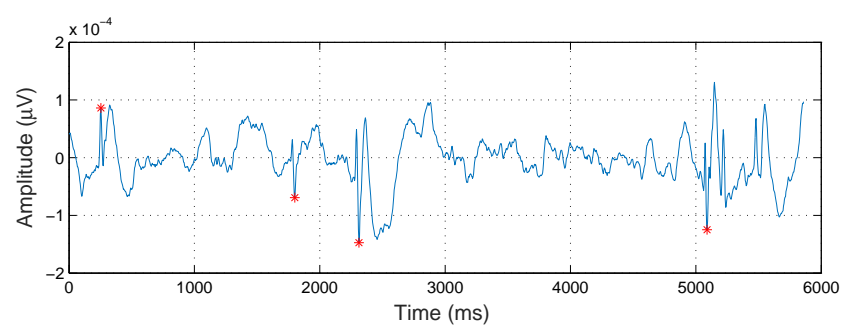

(a) Patient 2 in training dataset

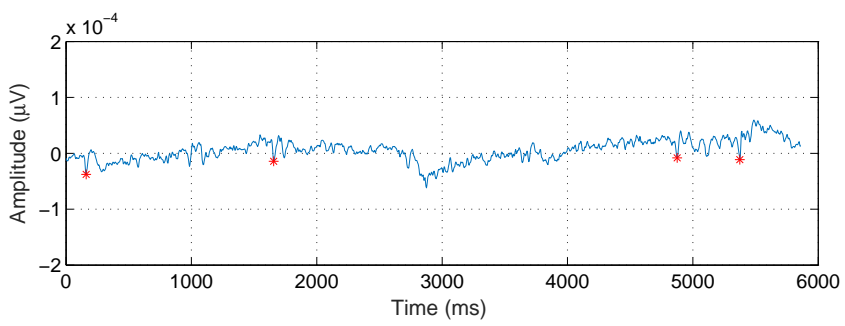

(b) Patient 11 in training dataset

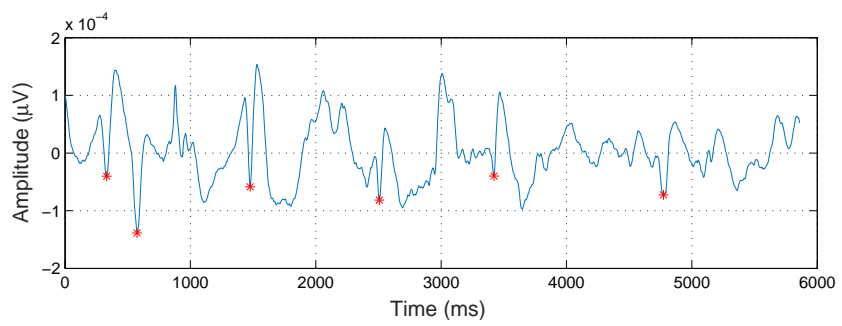

(c) Patient 3 in testing dataset

Figure 2. Some epileptic spikes in our EEG data set.

curve, going up and then down immediately [20]. Spikes appear irregularly and may manifest as independent or combined forms. They are often recorded in areas close to the impaired area of the brain and 


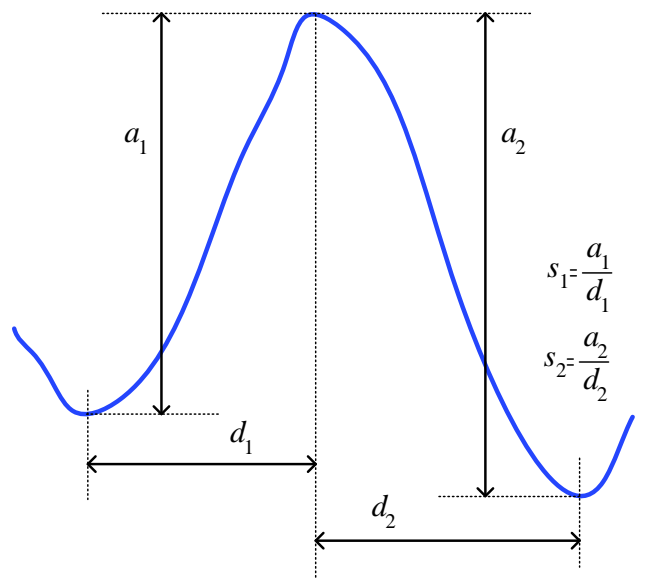

Figure 3. Six features parameterizing a significant peak [18] as inputs to the perceptron network in the preprocessing stage: First Half Wave Amplitude $\left(a_{1}\right)$, First Half Wave Duration $\left(d_{1}\right)$, Second Half Wave Amplitude $\left(a_{2}\right)$, Second Half Wave Duration $\left(d_{2}\right)$, First Half Wave Slope $\left(s_{1}\right)$ and Second Half Wave Slope $\left(s_{2}\right)$.

usually appear before a slow wave which lasts from 150 to $350 \mathrm{~ms}$ [19]. The morphology of spikes is diverse and complex depending on the patient and EEG recording conditions. Some epileptic spikes from our own data set are shown in Figure 2. It is not rare that different neurologists give different opinions about the appearances of the spikes on the same EEG signal. As such, evaluation results not only depend on the complexity of the EEG signals themselves but also on the level of expertise of the neurologists.

Since an epileptic spike has the shape of a peaky signal, to detect epileptic spikes accurately it is useful for the system in the preliminary stage to recognize all the peaks. In our system, a sample value is compared with its two nearest neighbors. If the sample value is largest, it is defined as a positive peak, and if the sample value is smallest, it is then defined as a negative peak.

After peak detection, the small peaks which are certainly not epileptic candidates are recognized as negligible peaks and removed based on the following threshold criteria: the distance between a pair of adjacent peaks is smaller than the distances between pairs of adjacent peaks right before and after. That is, given that peaks $p_{i-1}, p_{i}, p_{i+1}, p_{i+2}$ appear at times $t_{i-1}, t_{i}, t_{i+1}, t_{i+2}$, if the distance from $p_{i}$ to $p_{i+1}$ is smaller than that from $p_{i-1}$ to $p_{i}$ and that from $p_{i+1}$ to $p_{i+2}$, then $p_{i}$ and $p_{i+1}$ are removed. The duration of the peak (i.e., $d_{1}+d_{2}$ ) and the mean of its two relative amplitudes $\left(a_{1}, a_{2}\right)$ (as shown in Figure 3 ) are less than $20 \mathrm{~ms}$ and $17.5 \mu \mathrm{V}$, respectively. The reason for these thresholds is that the duration of epileptic spikes ranges from 20 to $70 \mathrm{~ms}$ and the amplitude of epileptic events normally lies between $20 \mu \mathrm{V}$ and $200 \mu \mathrm{V}$ [20]. This process makes it easier for the system to recognize epileptic spikes while a large number of peaks resulting from unwanted artefacts such as EMG (with small amplitude) are removed.

As mentioned earlier, neural network perceptrons are applied to classify the significant peaks into two groups: either non-epileptic spikes or possible epileptic

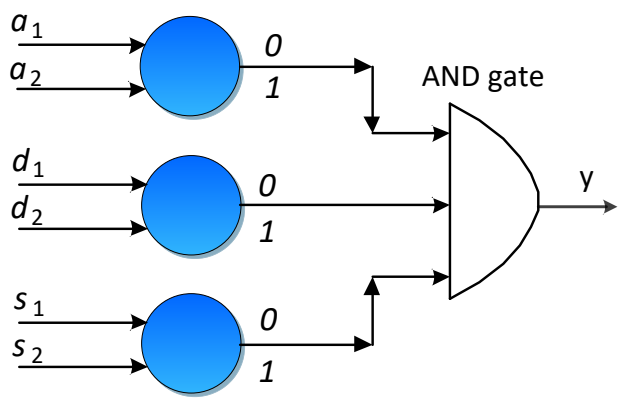

Figure 4. The proposed perceptron network for identifying possible spikes among significant EEG peaks. The inputs are six features determined as in Figure 3.

spikes. Similar to the work in [18], we use six features characterizing the amplitudes, durations, and slopes of a general epileptic spike as shown in Figure 3. They are First Half Wave Amplitude $\left(a_{1}\right)$, First Half Wave Duration $\left(d_{1}\right)$, Second Half Wave Amplitude $\left(a_{2}\right)$, Second Half Wave Duration $\left(d_{2}\right)$, First Half Wave Slope $\left(s_{1}\right)$ and Second Half Wave Slope $\left(s_{2}\right)$. These are the inputs to the perceptrons. Determining the number of perceptrons, the structure of the network and the proper procedure to train the perceptrons is non-trivial. Unlike [18], we propose to use a committee of three perceptrons (Figure 4), each is fed by two features (out of six) extracted from each significant peak. As shown in Figure 4, the output of each perceptron is either 0 or 1 and it is sent to an AND logic gate, so a significant peak is classified as a possible spike (belonging to group 2) if and only if the output of the AND logic gate equals 1 , meaning that all three perceptrons must return the same classification result of 1 .

\subsection{Feature Extraction}

Due to the nonstationary characteristics of EEG [21, 22], feeding the classifier with raw EEG signals often lead to poor classification results. Thus, in order to extract sufficient information from EEG signals, some time-frequency signal processing method should be utilized in advance. Various methods to represent signals in the joint time-frequency domain using timefrequency distributions were presented in [23]. The main difference among time-frequency methods is the way they handle the problem of uncertainty. Timefrequency methods have been used in various works for nonstationary signal analysis [24, 25] and for seizure detection [26, 27].

A frequently used time-frequency analysis technique is the wavelet transform which represents a signal by a set of well-defined basis functions known as wavelets [28, 29]. Wavelets are well localized in both time and frequency domains; that is, the wavelet distribution shows good resolution at a given small timefrequency region. Nowadays, wavelet transform have been successfully applied to the analysis of EEG signals as in [30-32], for epileptic spike detection as in [3, 3335] and seizure as in [36-39].

However, different choices of wavelets and scales could lead to different results and/or the degree of 


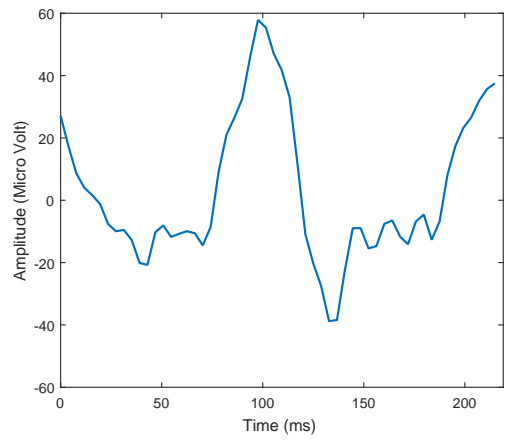

(a) Original spike in time

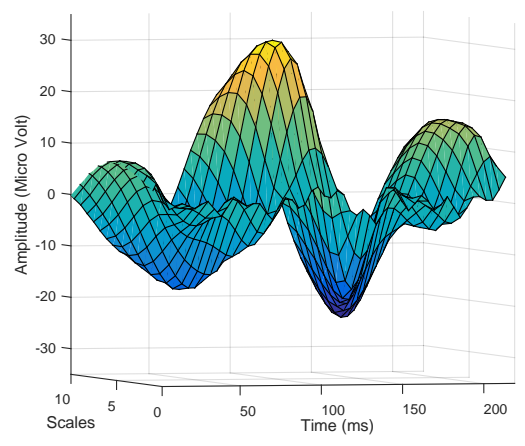

(b) 3D wavelet scalogram

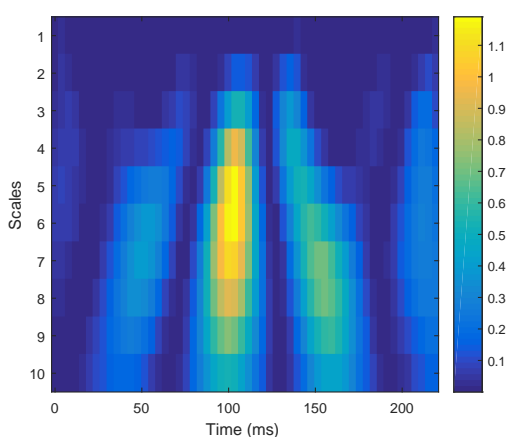

(c) Wavelet scalogram, 10 scales

Figure 5. Illustration of wavelet scales of a particular epileptic spike candidate. Original spike signal has a length of $219 \mathrm{~ms}$ (equivalent to 56 samples).

accuracy. In general, continuous wavelet transform is better than the discrete wavelet transform for timefrequency analysis of EEG signals because they allows us to examine a signal at any arbitrary wavelet scales (and, thus, frequencies) of interest while the latter can only provide information at specific discrete scales [23]. Taking advantages of wavelet transform, in our work, we use continuous wavelet transform for feature extraction.

Constructed from a single function $\psi(t)$, named mother wavelet or analyzing wavelet, wavelets are defined as

$$
\psi_{a, \tau}(t)=\frac{1}{\sqrt{a}} \psi\left(\frac{t-\tau}{a}\right),
$$

where $a$ and $\tau$ are dilation and translation factors, respectively.

Continuous wavelet transform (CWT) of a signal is defined as the correlations between the wavelet and the signal itself at different frequency scales and can be realized by the following formula:

$$
\operatorname{CWT}(x(t) ; a, \tau)=\frac{1}{\sqrt{a}} \int_{-\infty}^{\infty} x(t) \psi_{a, \tau}^{*}(t) d t,
$$

where $(\cdot)^{*}$ denotes the complex conjugate operator. In our work, the Mexican hat wavelet is selected as the mother wavelet due to its heuristic efficiency in exploiting nonstationary information of epileptic spikes [34]. The Mexican hat mother wavelet, $\psi(t)$, can be defined as

$$
\psi(t)=\frac{2}{\sqrt{3 \sigma} \pi^{1 / 4}}\left(1-\frac{t^{2}}{\sigma^{2}}\right) e^{-t^{2} / 2 \sigma^{2}},
$$

where $\sigma$ is a constant that has the same role as the standard deviation of a statistical distribution [40].

In [16], EEG signals with different lengths are analyzed by CWT. This process may yield errors in classification because the distribution of peaks in the segmented data is arbitrary and the threshold estimated by the background amplitude of the wavelet coefficients may not share consistent property. We recognize that the number of samples, the wavelet scales for decomposition and the wavelet coefficient selection for feature calculation should be carefully selected in order to achieve the best performance for the CWT.

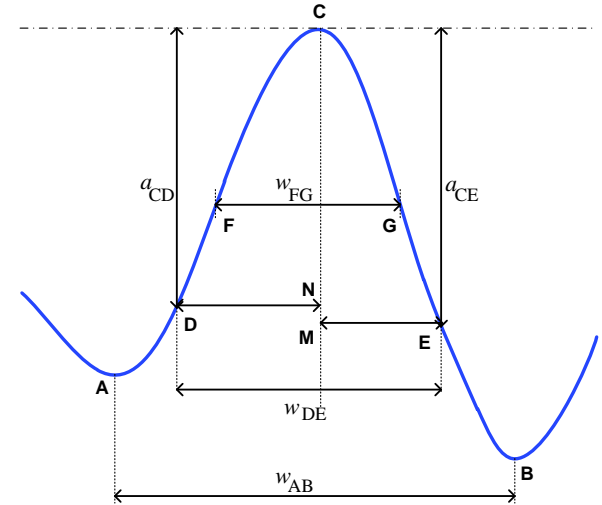

Figure 6. Wavelet features determined from a wavelet scale as inputs to the classifier stage [16].

In our experimental study, CWT is implemented over EEG segments of fixed length of 56 samples ( 25 samples before and 30 samples after each possible epileptic spike) at eight scales from 1 to 8 , obtained by varying the values of the dilation factor $a$. Spike information is found to be dominant in five highest scales (from scale 4 to 8). As shown in Figure 5, wavelet coefficients at those scales resemble the morphological characteristics of epileptic spike most.

These five scales are further utilized for calculating the features, as opposed to the 8 scales proposed in [16]. Specifically, seven types of features $\left(a_{\mathrm{CA}}, a_{\mathrm{CD}}, a_{\mathrm{CE}}, a_{\mathrm{CB}}\right.$, $\left.W_{\mathrm{FG}}, W_{\mathrm{DE}}, W_{\mathrm{AB}}\right)$ are computed for each scale coefficient set as characterized in Figure 6. More detailed explanation of the features can be found in [16]. After this step, those features are fed into the classifier stage for the task of epileptic spike classification.

\subsection{Classifier}

In a multi-stage system, the classifier is an integrated part in which the information after the feature extraction stage is utilized for determining whether the EEG peaks are candidate epileptic spikes. There are various types of classifiers: Support Vector Machines [41], Gaussian Mixture Model [42], Bayesian [43], Conditional Random Field [44], just to name a few. In our work, we use ANNs due to their well-known efficiency for classification [45]. 


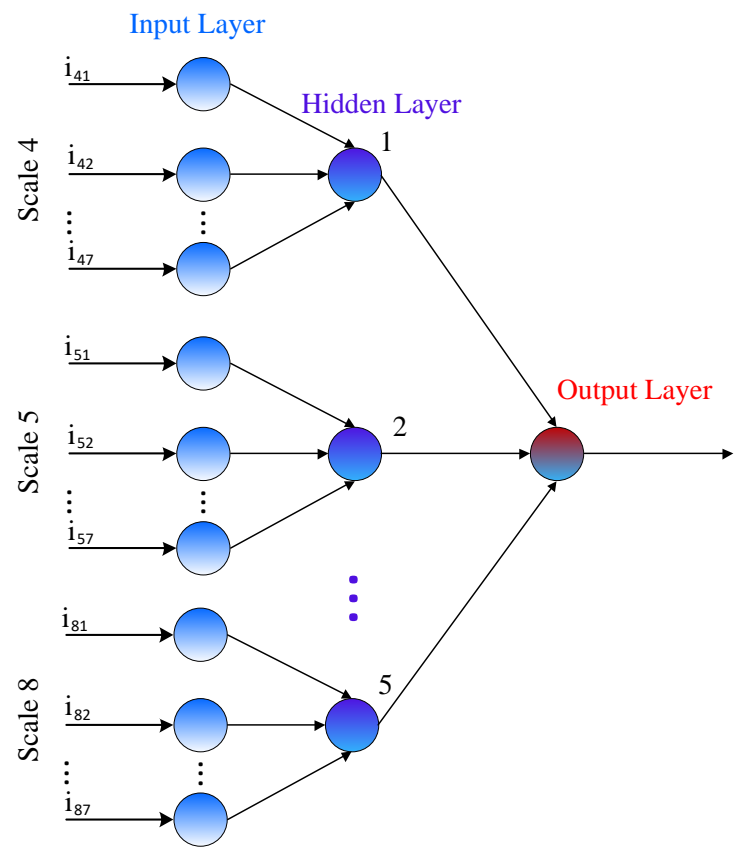

Figure 7. Structure of the selected ANN. There are 3 layers including an input layer, a hidden layer and an output layer. Specifically, the input layer includes 35 neurons. The hidden layer is composed of 5 neurons, and the output layer consists of one neuron.

An ANN is a bio-inspired mathematical model in which it mimics the structure of the human brain [46]. It is typically composed of three components including an input layer, one or more hidden layers and an output layer. Each layer is a set of neuronal units, called neurons, which are connected by weights to its previous and next layer units. The weight adjustment of network neuron connections are realized iteratively with a training scheme by minimizing the difference between desired output and actual output.

In this research, a "twice-learning" backpropagationbased method [16] is utilized as the training method for our three layer fully-connected ANNs. The structure of our ANN is illustrated in Figure 7. The inputs for each scale are seven features including $a_{\mathrm{CA}}, a_{\mathrm{CD}}, a_{\mathrm{CE}}, a_{\mathrm{CB}}$, $W_{\mathrm{FG}}, W_{\mathrm{DE}}, W_{\mathrm{AB}}$. We use five scales (from 4 to 8 ), so the number of nodes of our ANN is 41 .

There are two separate phases in the "twice-learning" training method. Spikes and non-spikes are used to train the ANN network. In the first phase, the ANN is trained by setting the desired output to be in the set $\{0,1\}$ in which 0 and 1 are corresponding to non-spike and spike, respectively. Feeding the testing data to an ANN yields the output with value in the range from 0 to 1 . In the second phase, the outputs $y$ from the first phase are categorized into two groups of spikes and non-spikes. The output samples after the first training phase are then sorted for minimum and maximum values $\left\{y_{\max }^{s}, y_{\min }^{s}\right\}$ for spike group and $\left\{y_{\max }^{n}, y_{\min }^{n}\right\}$ for non-spike group, and re-calculated as in [16] to yield

$$
\begin{aligned}
y^{s} & =0.45 \frac{y-y_{\min }^{s}}{y_{\max }^{s}-y_{\min }^{s}}+0.55, \\
y^{n} & =0.45 \frac{y-y_{\min }^{n}}{y_{\max }^{n}-y_{\min }^{n}} .
\end{aligned}
$$

After this step, all the spike output samples $y^{\mathcal{s}}$ have the corresponding desired outputs in the range $[0.55,1]$ and all the non-spike output samples $y^{n}$ have the desired outputs in the range [0,0.45].

Another ANN is trained with the new desired outputs. The same testing data as used in the first phase is then passed through the ANN for a regression process. The output returned by the network reflects the property of the corresponding EEG data input. After the classifier stage (i.e., the ANN), peaks having their outputs greater than a threshold will be sent to the expert system for further analysis.

In our experiment, the threshold for the ANN is set to 0.5 in order to achieve the best trade-off between specificity and sensitivity. In both phases, weights of the ANNs are updated using a backpropagation training scheme [46]. Often, a Receiver Operating Characteristic (ROC) curve is used to show the performance of a classifier at different thresholds. Figure 9 shows the performance of our ANN classifier, in which the above threshold value of 0.5 corresponds to the point on the curve that is closest to the top left corner. At that point, the performance is the best in the sense that the tradeoff between the specificity (SEN) and the sensitivity (SPE), as defined later in Section 4.2, is optimized $(\mathrm{SEN}=93 \% ; 1-\mathrm{SPE}=81 \%)$.

\subsection{Expert System}

In this section, we propose an expert system to remove pseudo (false) spikes which have not been identified previously. The expert system analyzes the spikes, previously marked by the classifier, to see if they are truly epileptic spikes, by exploiting the spatial and temporal contextual information. Specifically, there exists a neurological fact that an epileptic spike is often followed by slow waves rather than by another epileptic spike, and this is often called "spike and slow-wave complex" [19]. The slow-wave duration ranging from 150 to $350 \mathrm{~ms}$ indicates that there exist no more than two spikes in such a time interval [19].

The expert system is designed to then perform the following steps:

(a) Initiate a moving window with length of $350 \mathrm{~ms}$.

(b) Calculate the weighting parameter for every spike existing in the window as

$$
\rho=\frac{a_{1}+a_{2}}{2},
$$

where $a_{1}$ and $a_{2}$ are the First Half Wave Amplitude and Second Half Wave Amplitude of a spike, as shown in Figure 3.

(c) Remove the spikes whose weight $\rho$ fall below $k \rho_{\max }$, where $0 \leq k \leq 1$, and $\rho_{\max }$ is the local maximum of $\rho$ within the window of interest.

(d) Continue with the next window.

\section{Experimental Results ANd Analysis}

\subsection{Data Acquisition}

In this research, a 10-20 standard EEG recording system was used, providing 19-channel data, recorded 
Table I

Training Data Set

\begin{tabular}{ccccc}
\hline Pat. & Gen. & Age & Duration & Spikes \\
\hline 1 & Male & 21 & $23 \mathrm{~m} 57 \mathrm{~s}$ & 8 \\
2 & Male & 6 & $22 \mathrm{~m} 25 \mathrm{~s}$ & 635 \\
3 & Male & 9 & $11 \mathrm{~m} 24 \mathrm{~s}$ & 6 \\
4 & Male & 4 & $19 \mathrm{~m} 21 \mathrm{~s}$ & 8 \\
5 & Male & 15 & $22 \mathrm{~m} 0 \mathrm{~s}$ & 4 \\
6 & Male & 12 & $17 \mathrm{~m} 49 \mathrm{~s}$ & 22 \\
7 & Male & 72 & $15 \mathrm{~m} 26 \mathrm{~s}$ & 2 \\
8 & Male & 16 & $22 \mathrm{~m} 58 \mathrm{~s}$ & 11 \\
9 & Female & 16 & $20 \mathrm{~m} 14 \mathrm{~s}$ & 8 \\
10 & Female & 13 & $18 \mathrm{~m} 53 \mathrm{~s}$ & 5 \\
11 & Female & 20 & $14 \mathrm{~m} 32 \mathrm{~s}$ & 324 \\
12 & Female & 10 & $17 \mathrm{~m} 7 \mathrm{~s}$ & 2 \\
\hline
\end{tabular}

Pat. $=$ Patient, Gen.$=$ Gender

at a sampling rate of $256 \mathrm{~Hz}$. The duration for each recording varies from 6 to 28 minutes. Attached to the EEG system is a video recorder that can simultaneously track the movements of the patient, facilitating the neurologist task to discriminate the artifacts in the evaluation process.

We carried out measurements on patients who were clinically diagnosed to have epilepsy. There were 17 epileptic patients in total, with 11 males and 6 females. The data from 12 patients were used for system training (Table I) and the data from the other 5 patients (specifically, patient 5 has two durations: $5 \mathrm{a}$ and $5 \mathrm{~b}$ ) were used for evaluation of the performance of the proposed automatic detection system (Table II). The age of the patients ranges from 6 to 72 .

The average and bipolar montages were viewed during recording for verification but only signals obtained by the average montage were used for later evaluation. Due to the recording conditions, the EEG signals were highly contaminated by various types of artifacts. The epileptic spikes were recognized and labeled by one highly experienced neurologist from Vietnam National Children's Hospital.

After signal acquisition, the raw data were filtered by a band-pass filter with cut-off frequencies of 0.5 and $75 \mathrm{~Hz}$, and a notch filter of $50 \mathrm{~Hz}$ to remove the effect of the power-line frequency.

\subsection{Evaluation Metrics}

The sensitivity (SEN) and specificity (SPE) are used as the metrics for evaluating the performance of our proposed system; they are defined in [47] as follows:

$$
\begin{aligned}
& \mathrm{SEN}=\frac{\mathrm{TP}}{\mathrm{TP}+\mathrm{FN}} \times 100, \\
& \mathrm{SPE}=\frac{\mathrm{TN}}{\mathrm{TN}+\mathrm{FP}} \times 100,
\end{aligned}
$$

where true positive (TP) and true negative (TN) are the number of peaks that both the system and the neurologist agree to be, and, not to be epileptic spikes,
Table II

Testing Data Set

\begin{tabular}{ccccc}
\hline Pat. & Gen. & Age & Duration & Spikes \\
\hline 1 & Male & 9 & $11 \mathrm{~m} 24 \mathrm{~s}$ & 16 \\
2 & Male & 20 & $27 \mathrm{~m} 13 \mathrm{~s}$ & 1 \\
3 & Male & 11 & $16 \mathrm{~m} 16 \mathrm{~s}$ & 351 \\
4 & Female & 28 & $5 \mathrm{~m} 31 \mathrm{~s}$ & 12 \\
$5 \mathrm{a}$ & Female & 22 & $27 \mathrm{~m} 37 \mathrm{~s}$ & 19 \\
$5 \mathrm{~b}$ & Female & 22 & $27 \mathrm{~m} 37 \mathrm{~s}$ & 9 \\
\hline
\end{tabular}

Pat.$=$ Patient, Gen.$=$ Gender

respectively, the false positive (FP) is the number of peaks that the system labels as epileptic spikes but the neurologist considers as normal, and false negative (FN) is the number of peaks that the neurologist labels as epileptic spikes but the system does not recognize so.

To evaluate the system performance, the sensitivity is averaged across different records. We use four methods presented in [47], which give accurate characterisation of interictal spike detection algorithms. Denote by $T_{i}$ the duration of $i$-th record, $M_{i}$ the number of spikes marked by the neurologist, and $C_{i}$ the number of correctly detected spikes. Then, the sensitivity of the $i$-th record is given by $C_{i} / M_{i}$. The average sensitivity of $N$ records is calculated by four different methods as follows:

Arithmetic mean: $\mathrm{SEN}=\frac{1}{N} \sum_{i=1}^{N} \frac{C_{i}}{M_{i}}$

Time-weighted average: SEN $=\frac{1}{\sum_{i=1}^{N} T_{i}} \sum_{i=1}^{N} \frac{C_{i}}{M_{i}} T_{i}$

Total sensitivity average: SEN $=\frac{1}{\sum_{i=1}^{N} M_{i}} \sum_{i=1}^{N} C_{i}$

Time/event-weighted average: SEN $=\frac{1}{\sum_{i=1}^{N} \frac{T_{i}}{M_{i}}} \sum_{i=1}^{N} \frac{C_{i}}{M_{i}} \frac{T_{i}}{M_{i}}$

\subsection{Results}

In this section, the experimental results are reported graphically and statistically. Figure 8 illustrates the performance of the system stage by stage on an original EEG segment as in Figure 8(a).

The results suggest that the number of spikes are significantly reduced after each step. The system starts the screening process with a very simple function, identifying all the peaks over the data segment (Figure $8(\mathrm{~b}))$. However, not all the peaks are candidate epileptic spikes and many of them are "negligible" and absolutely not the ones we are seeking for (Figure 8(c)). Those negligible peaks are totally removed in the next step of the preprocessing stage (Figure $8(\mathrm{~d})$ ). In the last step of the preprocessing stage, three perceptrons are 


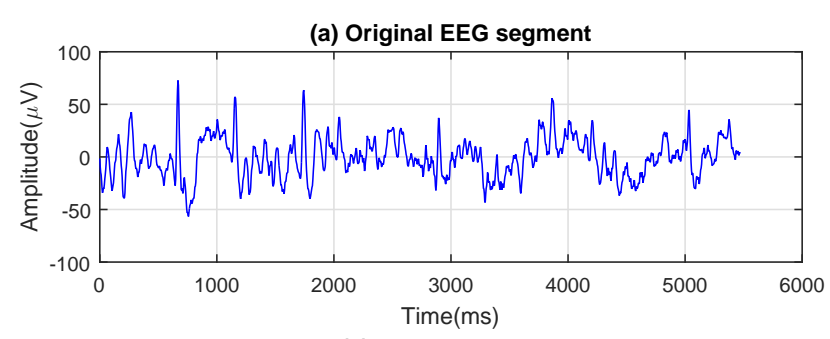

(c) Negligible peaks

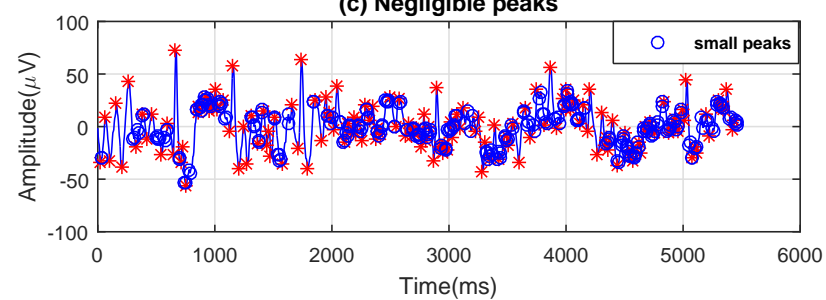

(e) Possible spikes

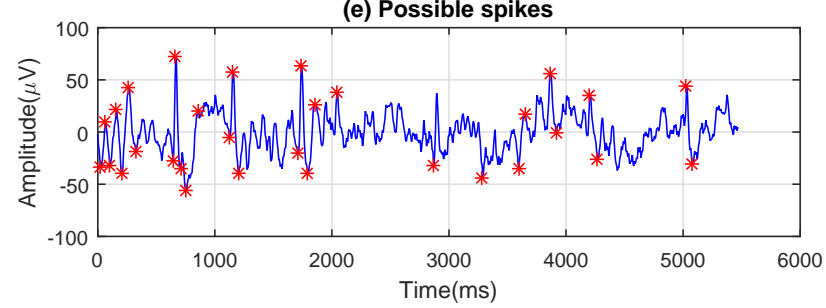

(b) All peaks

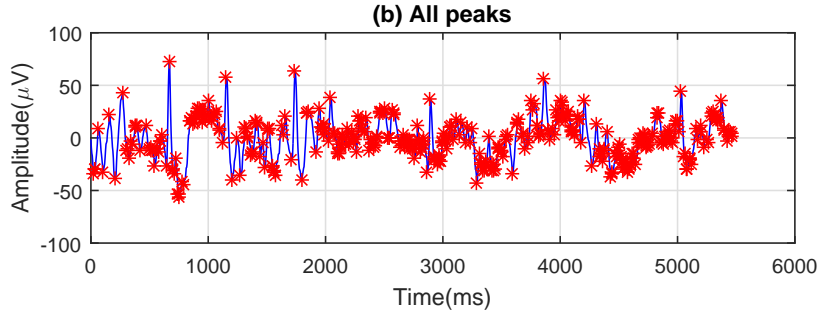

(d) Remaining peaks

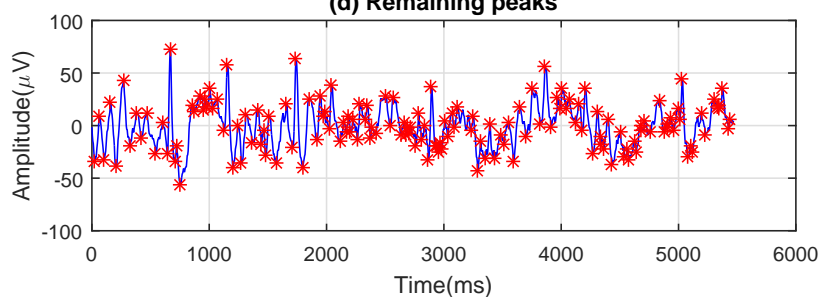

(f) Epileptic spikes

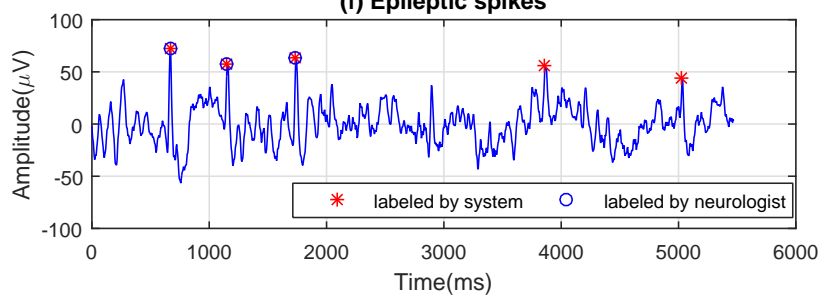

Figure 8. Illustrative results obtained by the proposed spike detection system. The original EEG segment (a) has the length of 5.86 seconds (equivalent to 1500 samples). Preprocessing the original signal results in possible epileptic spikes (e). Combination of the two classifiers, the ANN and the expert system, in identifying epileptic spikes (f), which also shows the comparison between the spikes detected by the system and the epileptic spikes labeled by the neurologist.

utilized efficiently for removing a large portion of the peaks (Figure 8(e)). After the preprocessing stage, all of the spike candidates for epilepsy are recognized and passed to the next stage of classifying by an ANN.

The task of the ANN becomes harder because the morphological characteristics of the candidate spikes are so analogous. That is why a careful "twice learning" scheme is used to greatly facilitate the system in removing non-epileptic spikes [16].

Last but not least, a simple expert system is proposed to exclude pseudo-spikes. In our data, the value of $k$ was set to be 0.95 for the best performance of the expert system. This value was obtained emperically via preliminary experiments, which are not discussed here.

The final result is given in Figure 8(f), which also shows the difference between the results returned by the system and the neurologist. It is easily recognized that all the epileptic spikes labeled by the neurologist were identified by the proposed multi-stage system. However, not all the spikes detected by the system are truly epileptic according to the neurologist judgment.

The performance of the proposed system in terms of quantitative analysis also shows that the number of candidate epileptic spikes were naturally reduced after each step. Specifically, there is a significant decrease in the number of peaks/spikes after the preprocessing and ANN stages. The expert system stage (the overall result) slightly decreases the number of spikes; clearly this depends on the different data and if there exist many pseudo-spikes near an epileptic spike or not.

In the feature extraction stage, all 10 wavelet scales of a candidate epileptic spike are shown in Figure 5. By ex-
Table III

System Detection Results by Stages

\begin{tabular}{lrrrrr}
\hline Patient & \multicolumn{3}{c}{ Preprocessing } & ANN & ES \\
\hline & all & sig. & pos. & epil. & sure \\
\hline 1 & 1795728 & 1269227 & 36885 & 7160 & 6276 \\
2 & 1266464 & 477319 & 6975 & 945 & 840 \\
3 & 3701808 & 2388564 & 36361 & 4697 & 4357 \\
4 & 2540528 & 1829433 & 24942 & 3913 & 3403 \\
$5 \mathrm{a}$ & 1120848 & 607211 & 13251 & 3827 & 3144 \\
$5 \mathrm{~b}$ & 1224816 & 979726 & 14226 & 4589 & 3683 \\
\hline
\end{tabular}

sig. $=$ significant, pos. $=$ possible, epil. $=$ epileptic

Table IV

Sensitivity and Specificity Calculations

\begin{tabular}{lrrrrrrrr}
\hline Pat. & Dur. & Spk & TP & FP & FN & TN & SEN & SPE \\
\hline 1 & $11 \mathrm{~m} 24 \mathrm{~s}$ & 16 & 14 & 6262 & 2 & 30609 & 87.50 & 83.01 \\
2 & $27 \mathrm{~m} 13 \mathrm{~s}$ & 1 & 1 & 839 & 0 & 6135 & 100.00 & 87.97 \\
3 & $16 \mathrm{~m} 16 \mathrm{~s}$ & 351 & 323 & 4034 & 28 & 32004 & 92.02 & 88.80 \\
4 & $5 \mathrm{~m} 31 \mathrm{~s}$ & 12 & 12 & 3391 & 0 & 21539 & 100.00 & 86.40 \\
$5 \mathrm{a}$ & $27 \mathrm{~m} 37 \mathrm{~s}$ & 19 & 18 & 3126 & 1 & 10107 & 94.74 & 76.37 \\
$5 \mathrm{~b}$ & $27 \mathrm{~m} 37 \mathrm{~s}$ & 9 & 9 & 3674 & 0 & 10543 & 100.00 & 74.16 \\
\hline
\end{tabular}

Pat. $=$ Patient, Dur. $=$ Duration, Spk $=$ number of spikes.

amining various candidate epileptic spikes, we choose to use only scales from 4 to 8 , which reflects well the morphological property of the original epileptic spikes.

Tables III show the number of spikes detected by the proposed multistage system, stage by stage, evaluated 
Table V

Sensitivity Averaging Methods

\begin{tabular}{cc}
\hline Methods & SEN \\
\hline Arithmetic mean & 96.75 \\
Time-weighted average & 95.99 \\
Total sensitivity average & 92.64 \\
Time/event-weighted average & 99.38 \\
\hline STD & $\mathbf{2 . 7 7}$ \\
\hline
\end{tabular}

on the testing data set of 5 patients. As a result, the sensitivity and specificity were calculated as shown in Table IV. It can be seen that the sensitivity is very different among the patients (the smallest is $87.5 \%$ and largest is $100 \%$ ). The main reason is the difference in the epileptic characteristics among the patients that are clearly seen in Table IV via the recording time (Dur.) and the number of epileptic events (Spikes). For example, patient \#2 has 1 spike labeled by the neurologist for a recording time of $27 \mathrm{~m} 13 \mathrm{~s}$ while patient \#3 has 351 spikes for $16 \mathrm{~m} 16 \mathrm{~s}$. Therefore, it is most appropriate to use the time/event-weighted average method for evaluating the sensitivity for the underlying data, as compared to the three other methods. Accordingly, the sensitivity of the proposed system is $99.38 \%$, as shown in Table V. Comparing the results between Table IV and Table VI, we can see that the expert system removes pseudo (false) spikes, thus significantly increasing the system specificity.

As briefly mentioned in Section 3.3, the ROC curve is a powerful tool for measuring the performance of a classifier, and has been successfully applied in various applications such as biomedical research, data science or machine learning. The curve is created by plotting the sensitivity (which is the true positive rate) against the false positive rate (equal to $1-\mathrm{SPE}$ ) at different thresholds. Overall, the area under the ROC curve (AUC) allows us to estimate how efficiently a classifier is. The AUC value lying between 0.9 and 1 indicates an excellent classifier, between 0.8 and 0.9 shows a good classifier, between 0.7 and 0.8 presents a fair classifier, and between 0.6 and 0.7 denotes a poor classifier.

The ROC curve of our classifier is visualized in Figure 9. The blue curve shows the ROC on the training data set and the red curve shows the ROC on the testing data set of our classifier. As shown in Figure 9, the AUC of the training data set is 0.972 and the AUC of the testing data set is 0.945 . This means that the performance of our ANN classifier is excellent on all data set.

\section{Discussions}

The evaluation and comparison of different automatic spike detection systems are not easy tasks since the data sets applied for different systems are not the same [3]. It means that there is a chance for a system to have good performance on a certain data set, but still produce a very different performance on another data set. An-
Table VI

Results, without Using the Expert System

\begin{tabular}{lrrrrrrrr}
\hline Pat. & Dur. & Spk & TP & FP & FN & TN & SEN & SPE \\
\hline 1 & $11 \mathrm{~m} 24 \mathrm{~s}$ & 16 & 14 & 7146 & 2 & 29725 & 87.50 & 80.60 \\
2 & $27 \mathrm{~m} 13 \mathrm{~s}$ & 1 & 1 & 944 & 0 & 6030 & 100.00 & 86.46 \\
3 & $16 \mathrm{~m} 16 \mathrm{~s}$ & 351 & 323 & 4374 & 28 & 31664 & 92.02 & 87.86 \\
4 & $5 \mathrm{~m} 31 \mathrm{~s}$ & 12 & 12 & 3901 & 0 & 21029 & 100.00 & 84.35 \\
$5 \mathrm{a}$ & $27 \mathrm{~m} 37 \mathrm{~s}$ & 19 & 19 & 3808 & 0 & 9424 & 100.00 & 71.22 \\
$5 \mathrm{~b}$ & $27 \mathrm{~m} 37 \mathrm{~s}$ & 9 & 9 & 4580 & 0 & 9637 & 100.00 & 67.78 \\
\hline
\end{tabular}

Pat. $=$ Patient, Dur. $=$ Duration, Spk $=$ number of spikes.

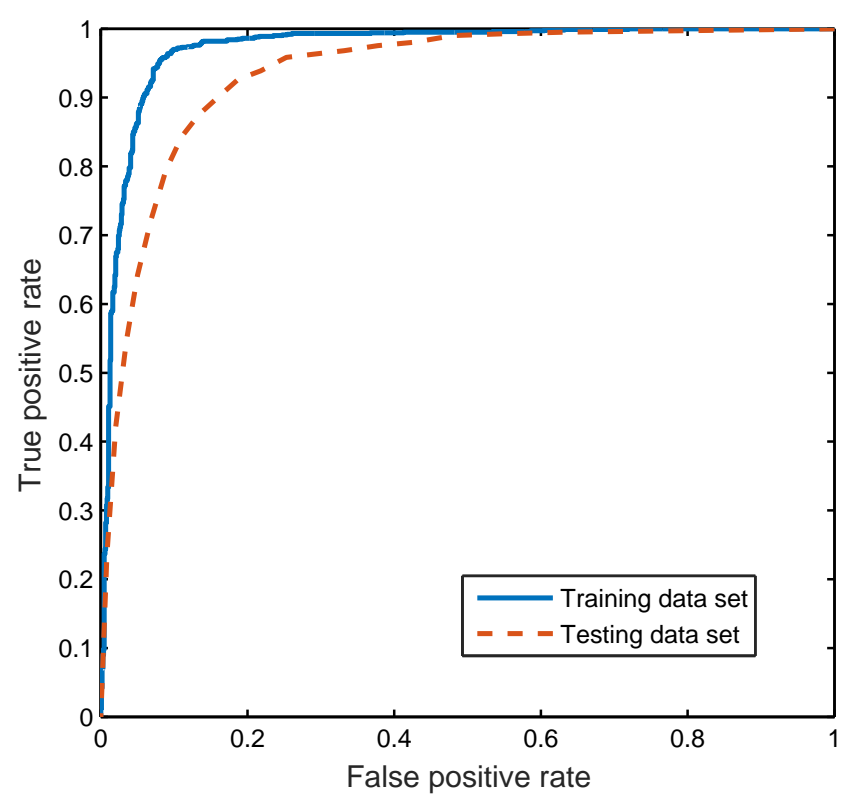

Figure 9. ROC curves. The solid line shows the ROC on the training data set and the dash line shows the ROC on the testing data set of the ANN classifier. The AUC of the solid line is 0.972 and of the dash one is 0.945 .

other problem is that different neurologists often have different perspectives on epileptic spikes. In addition, the performance of an automatic spike detection system relies on the characteristics of the recording. Seemingly, if the EEG recording contains more epileptic events the performance is better [48].

In fact, there is currently no perfect independent engineering method at detecting spikes, so a good system must combine various methods at multiple stages based on information about shape, frequency, time and context in which spikes appear [16, 49].

Our proposed system is trained and tested on EEG recording data from patients who have been diagnosed to have epilepsy. The averaged sensitivity of the system is $99.38 \%$, indicating that the system is capable of detecting epileptic spikes efficiently.

Meanwhile, as mentioned in Section 4 the results returned by the system on EEG data from all other patients are stable and could be used for our initial purpose of providing an assisting tool for neurologists in epileptic diagnosis. The fact that our system performs well in some cases while not so well in other cases (rarely) is explainable. That is, our proposed 
multi-stage system consists of multiple perceptrons and ANNs which are well-known for their universal approximate capability. Thus, the system also inherits that universal approximation characteristic. Furthermore, as mentioned previously, the recording conditions in Vietnam are not standardized so our data contamination level may vary significantly and thus the system performance may be poor when the contamination level in the recording is high.

In some previous works, perfect SENs [18] (on EEG data of several patients) or Accuracy [50] are reported for their system performances. Those results might not be applicable to a larger unseen data.

The multistage system for automatic detection and classification of epileptiform in [16] consists of adaptive filtering, wavelet transform, artificial neural network, and expert system. EEG signals are analyzed by the wavelet transform at 32 scales and the detection of epileptic spikes is performed on the extracted features, as inputs to the classifier stage, from the wavelet coefficients of 8 lowest scales. They use a very complex expert system which requires a lot of information. The AUC is around 0.95 and the SEN for detection spikes, sharp waves, and complexes is $92.4 \%$.

In [18], a three-stage system was proposed for automatic detection of epileptiform transient (ET) based on artificial neural networks. In its pre-classification stage, they use two discrete perceptrons fed by six features to classify EEG peaks. In the second stage, possible ETs and possible non-ETs are classified by a nonlinear artificial neural network. In the third stage, multichannel information is integrated to identify an epileptiform event (EV) by the electroencephalographers. The best performance of their system is based on a radial basis support vector machine (RB-SVM) providing an average sensitivity of $89.1 \%$. In this work, the AUC value was not given.

The work in [51] using a new technique, called convolutional neural network (CNN), did not apply any feature extraction method. The value of AUC achieved in [51] is 0.947 but the SEN value is not provided.

Compared to other works, our proposed system uses a committee of three perceptrons, appropriate mother wavelet and wavelet scales by which the wavelet transform is implemented only at eight scales and the features are calculated from the coefficients of only 5 scales (from scale 4 to scale 8 ). Thus, the implementation of the proposed system requires less computational power. The sensitivity of the proposed system is $99.38 \%$ and the AUC is around 0.947, which is as high as the AUC reported in [51].

The EEG data in our experiment were labeled by only one neurologist and then used as the golden standard to train our system. If the neurologist did not correctly recognize an epileptic spike, it is likely that the system repeats the misjudgment. Thus, the performance of the current system could be improved when more neurologists are involved in the expert judgement process.

The functionality of the expert system, which is added as the last stage of the multistage system, is to recognize pseudo-spikes out of epileptic spike can- didates identified in previous stages. An appropriate expert system should be selected in such a way that it does not eliminate true epileptic spikes and, at the same time, recognizes as many pseudo-spikes as possible. That selection is non-trivial. In addition, the performance of the expert system depends largely on the selection of thresholding value $k \rho_{\max }$. A lower threshold allows more spikes to be detected by the system, and therefore increases true positive but also increases false positive. Thus, there lies always a trade-off between sensitivity and specificity. In our data, the threshold is selected to be 0.95 for the best performance of the expert system. Note that the expert system proposed in [16] is rather complex, including various types of information in the decision making process. Our expert system only focuses on the use of morphological information.

In terms of morphological characteristic of epileptic spikes, Boos et al. [19] reported that on the occurrence of an epileptic event, there is always a slow wave to follow a spike. However, it is not always true in terms of neurological perspective based on our neurologist experience. In fact, there could be appearances of multiple spikes in a single epileptic event. Fortunately, we do not have to handle that (rare) phenomenon (which certainly leads to high rate of false recognition of epileptic spikes by our expert system) in our experimentation thanks to the way we selected epileptic patients for EEG recording. Accordingly, the too severe epileptic patients, whose EEG signals are likely to contain multiple epileptic spikes, were not selected.

\section{Conclusions}

In summary, this paper introduces a novel multistage system for epileptic spike detection as an assistant tool for epileptic diagnosis, especially useful in Vietnam where recording conditions are limited. The system consists of four main stages: preprocessing, feature extraction, classifier and expert system. At each stage, different engineering techniques, from basic signal processing methods in spatial domain to advanced timefrequency and machine learning ones, have been employed. Specifically, spectral filtering is integrated into the preprocessing stage, wavelet transform is implemented for the feature extraction, different neural networks are utilized at the classifier stage, and a novel set of rules are built in the expert system. The system has been successfully implemented and validated on real EEG data of epileptic patients. further, in the experiments conducted, the EEG data that were available contain artefacts and the proposed system was capable of detecting the spikes even in the presence of contaminated data.

The results also indicate that the epileptic spike amplitudes were not always stronger than the background EEG signal. In fact, sometimes epileptic spikes were not so distinguishable due to its low voltage amplitude. Our expert system might have made mis-judgment in these cases. This is a limitation to the entire system and need to be investigated carefully in future work. 
The comparison of our proposed method with other multistage methods [16-18, 51, 52] is subject to a subsequent study, using the same data set. Additionally, extension to multi-channel analysis, taking into account the spike spatial correlation, is expected to improve the classification (see, e.g. $[1,26]$ ). Finally, one can expect further improvements with the methods presented in $[53,54]$ as there is a comparison between wavelets and quadratic TFDs for classification.

\section{ACKNOWLedgments}

The authors would like to thank Dr. Hoang Cam Tu from Vietnam National Children's Hospital for recording and interpreting the EEG data. This work was supported by Project QG.10.40 from Vietnam National University, Hanoi.

\section{REFERENCES}

[1] K. Indiradevi, E. Elias, P. Sathidevi, S. Dinesh Nayak, and K. Radhakrishnan, "A multi-level wavelet approach for automatic detection of epileptic spikes in the electroencephalogram," Computers in Biology and Medicine, vol. 38, no. 7, pp. 805-816, 2008.

[2] J. Gotman, "Automatic detection of seizures and spikes," Journal of Clinical Neurophysiology, vol. 16, no. 2, pp. 130$140,1999$.

[3] S. B. Wilson and R. Emerson, "Spike detection: A review and comparison of algorithms," Clinical Neurophysiology, vol. 113, no. 12, pp. 1873-1881, 2002.

[4] A. T. Tzallas, M. G. Tsipouras, D. G. Tsalikakis, E. C. Karvounis, L. Astrakas, S. Konitsiotis, and M. Tzaphlidou, "Automated epileptic seizure detection methods: A review study," Epilepsy-histological, electroencephalographic and psychological aspects, edited by D. Stevanovic, pp. 75-98, 2012.

[5] P. Y. Ktonas and J. R. Smith, "Quantification of abnormal EEG spike characteristics," Computers in Biology and Medicine, vol. 4, no. 2, pp. 157-163, 1974.

[6] J. Gotman and P. Gloor, "Automatic recognition and quantification of interictal epileptic activity in the human scalp EEG," Electroencephalography and Clinical Neurophysiology, vol. 41, pp. 513-529, 1976.

[7] G. Pfurtscheller and G. Fischer, "A new approach to spike detection using a combination of inverse and matched filter techniques," Electroencephalography and clinical neurophysiology, vol. 44, no. 2, pp. 243-247, 1978.

[8] J. Gotman and L. Wang, "State-dependent spike detection: concepts and preliminary results," Electroencephalography and Clinical Neurophysiology, vol. 79, no. 1, pp. 11-19, 1991.

[9] J. Gotman, "Automatic recognition of epileptic seizures in the EEG," Electroencephalography and Clinical Neurophysiology, vol. 54, no. 5, pp. 530-540, 1982.

[10] A. Ossadtchi, S. Baillet, J. Mosher, D. Thyerlei, W. Sutherling, and R. Leahy, "Automated interictal spike detection and source localization in magnetoencephalography using independent components analysis and spatiotemporal clustering," Clinical Neurophysiology, vol. 115, no. 3, pp. 508-522, 2004.

[11] G. Xu, J. Wang, Q. Zhang, S. Zhang, and J. Zhu, "A spike detection method in EEG based on improved morphological filter," Computers in Biology and Medicine, vol. 37, no. 11, pp. 1647-1652, 2007.

[12] Ö. Özdamar and T. Kalayci, "Detection of spikes with artificial neural networks using raw EEG," Computers and Biomedical Research, vol. 31, no. 2, pp. 122-142, 1998.
[13] C. C. Pang, A. R. Upton, G. Shine, and M. V. Kamath, “A comparison of algorithms for detection of spikes in the electroencephalogram," IEEE Transactions on Biomedical Engineering, vol. 50, no. 4, pp. 521-526, 2003.

[14] T.-W. Shen, X. Kuo, and Y.-L. Hsin, "Ant K-Means Clustering Method on Epileptic Spike Detection," in Proceedings of the Fifth International Conference on Natural Computation (ICNC'09), vol. 6. IEEE, 2009, pp. 334-338.

[15] N. Acır and C. Güzeliş, "Automatic spike detection in EEG by a two-stage procedure based on support vector machines," Computers in Biology and Medicine, vol. 34, no. 7, pp. 561-575, 2004.

[16] H. S. Liu, T. Zhang, and F. S. Yang, "A multistage, multimethod approach for automatic detection and classification of epileptiform EEG," IEEE Transactions on Biomedical Engineering, vol. 49, no. 12, pp. 1557-1566, 2002.

[17] H. Hamid and B. Boashash, "A time-frequency approach for EEG spike detection," Iranica Journal of Energy $\mathcal{E}$ Environment, vol. 2, no. 4, pp. 390-395, 2011.

[18] N. Acır, İ. Öztura, B. Baklan, and C. Güzeliş, “Automatic Detection of Epileptiform Events in EEG by a ThreeStage Procedure Based on Artificial Neural Networks," IEEE Transactions on Biomedical Engineering, vol. 52, no. 1, pp. 30-40, 2005.

[19] C. F. Boos, F. M. d. A. G. R. Scolaro, and V. P. Maria do Carmo, Automatic Detection of Paroxysms in EEG Signals using Morphological Descriptors and Artificial Neural Networks. INTECH Open Access Publisher, 2011.

[20] E. Niedermeyer and F. L. da Silva, Electroencephalography: basic principles, clinical applications, and related fields. Lippincott Williams \& Wilkins, 2005.

[21] J. S. Barlow, "Methods of analysis of nonstationary eegs, with emphasis on segmentation techniques: a comparative review." Journal of Clinical Neurophysiology, vol. 2, no. 3, pp. 267-304, 1985.

[22] H. Azami, S. Sanei, K. Mohammadi, and H. Hassanpour, "A hybrid evolutionary approach to segmentation of non-stationary signals," Digital Signal Processing, vol. 23, no. 4, pp. 1103-1114, 2013.

[23] B. Boashash, Time-frequency signal analysis and processing: a comprehensive reference. Academic Press, 2015.

[24] J. P. Amezquita-Sanchez and H. Adeli, "A new musicempirical wavelet transform methodology for timefrequency analysis of noisy nonlinear and non-stationary signals," Digital Signal Processing, vol. 45, pp. 55-68, 2015.

[25] V. Sucic, N. Saulig, and B. Boashash, "Analysis of local time-frequency entropy features for nonstationary signal components time supports detection," Digital Signal Processing, vol. 34, pp. 56-66, 2014.

[26] B. Boashash and G. Azemi, "A review of time-frequency matched filter design with application to seizure detection in multichannel newborn EEG," Digital Signal Processing, vol. 28, pp. 28-38, 2014.

[27] B. Boashash, N. A. Khan, and T. Ben-Jabeur, "Timefrequency features for pattern recognition using highresolution TFDs: A tutorial review," Digital Signal Processing, vol. 40, pp. 1-30, 2015.

[28] O. Rioul and M. Vetterli, "Wavelets and signal processing," IEEE Signal Processing Magazine, vol. 8, no. 4, pp. 14-38, 1991.

[29] I. Daubechies, Ten lectures on wavelets. SIAM, 1992, vol. 61.

[30] S. J. Schiff, A. Aldroubi, M. Unser, and S. Sato, "Fast wavelet transformation of EEG," Electroencephalography and Clinical Neurophysiology, vol. 91, no. 6, pp. 442-455, 1994.

[31] T. Kalayci and Ö. Özdamar, "Wavelet preprocessing for automated neural network detection of EEG spikes," Engineering in Medicine and Biology Magazine, IEEE, vol. 14, no. 2, pp. 160-166, 1995.

[32] V. J. Samar, A. Bopardikar, R. Rao, and K. Swartz, "Wavelet analysis of neuroelectric waveforms: a concep- 
tual tutorial," Brain and language, vol. 66 , no. 1, pp. 7-60, 1999.

[33] Z. Nenadic and J. W. Burdick, "Spike detection using the continuous wavelet transform," IEEE Transactions on Biomedical Engineering, vol. 52, no. 1, pp. 74-87, 2005.

[34] M. Latka, Z. Was, A. Kozik, and B. J. West, "Wavelet analysis of epileptic spikes," Physical Review E, vol. 67, no. 5, p. 052902, 2003.

[35] R. Q. Quiroga, Z. Nadasdy, and Y. Ben-Shaul, "Unsupervised spike detection and sorting with wavelets and superparamagnetic clustering," Neural Computation, vol. 16, no. 8, pp. 1661-1687, 2004.

[36] Y. Khan and J. Gotman, "Wavelet based automatic seizure detection in intracerebral electroencephalogram," Clinical Neurophysiology, vol. 114, no. 5, pp. 898908, 2003.

[37] S. Ghosh-Dastidar, H. Adeli, and N. Dadmehr, "Mixedband wavelet-chaos-neural network methodology for epilepsy and epileptic seizure detection," IEEE Transactions on Biomedical Engineering, vol. 54, no. 9, pp. 15451551, 2007.

[38] H. Adeli, S. Ghosh-Dastidar, and N. Dadmehr, "A wavelet-chaos methodology for analysis of eegs and eeg subbands to detect seizure and epilepsy," IEEE Transactions on Biomedical Engineering, vol. 54, no. 2, pp. 205-211, 2007.

[39] E. D. Übeyli, "Combined neural network model employing wavelet coefficients for eeg signals classification," Digital Signal Processing, vol. 19, no. 2, pp. 297-308, 2009.

[40] X. Mi, H. Ren, Z. Ouyang, W. Wei, and K. Ma, "The use of the Mexican Hat and the Morlet wavelets for detection of ecological patterns," Plant Ecology, vol. 179, no. 1, pp. $1-19,2005$.

[41] C. Cortes and V. Vapnik, "Support-vector networks," Machine learning, vol. 20, no. 3, pp. 273-297, 1995.

[42] C. A. Bouman, M. Shapiro, G. Cook, C. B. Atkins, and H. Cheng, "CLUSTER: An unsupervised algorithm for modeling Gaussian mixtures," 1997.

[43] C. M. Bishop and N. M. Nasrabadi, Pattern recognition and machine learning. springer New York, 2006, vol. 1.

[44] J. Lafferty, A. McCallum, and F. C. Pereira, "Conditional random fields: Probabilistic models for segmenting and labeling sequence data," in Proceedings of the Eighteenth International Conference on Machine Learning, San Francisco, CA, USA, 2001, pp. 282-289.

[45] T. M. Mitchell, "Machine learning. 1997," Burr Ridge, IL: McGraw Hill, vol. 45, no. 37, pp. 870-877, 1997.

[46] D. E. Rumelhart, G. E. Hintont, and R. J. Williams, "Learning representations by back-propagating errors," Nature, vol. 323, no. 6088, pp. 533-536, 1986.

[47] Casson, A. J., E. Luna, and E. Rodriguez-Villegas, "Performance metrics for the accurate characterisation of interictal spike detection algorithms," Journal of neuroscience methods, vol. 177, no. 2, pp. 479-487, 2009.

[48] J. D. Frost, "Automatic Recognition and Characterization of Epileptiform Discharges in the Human EEG," Clinical Neurophysiology, no. 231-249, 1985.

[49] A. A. Dingle, R. D. Jones, G. Carroll, and W. Fright, "A multistage system to detect epileptiform activity in the EEG," IEEE Transactions on Biomedical Engineering, vol. 40, no. 12 , pp. $1260-1268,1993$.

[50] C. A. Lima, A. L. Coelho, and M. Eisencraft, "Tackling EEG signal classification with least squares support vector machines: A sensitivity analysis study," Computers in Biology and Medicine, vol. 40, no. 8, pp. 705-714, 2010.

[51] A. R. Johansen, J. Jin, T. Maszczyk, J. Dauwels, S. S.
Cash, and M. B. Westover, "Epileptiform spike detection via convolutional neural networks," in Proceedings of the IEEE International Conference on Acoustics, Speech and Signal Processing (ICASSP). IEEE, 2016, pp. 754-758.

[52] B. Boashash, H. Barki, and S. Ouelha, "Performance evaluation of time-frequency image feature sets for improved classification and analysis of non-stationary signals: Application to newborn EEG seizure detection," RnowledgeBased Systems, vol. 132, pp. 188-203, 2017.

[53] B. Boashash and S. Ouelha, "Efficient software platform TFSAP 7.1 and Matlab package to compute timefrequency distributions and related time-scale methods with extraction of signal characteristics," SoftwareX, 2017.

[54] — - "Designing high-resolution time-frequency and time-scale distributions for the analysis and classification of non-stationary signals: a tutorial review with a comparison of features performance," Digital Signal Processing, 2017.

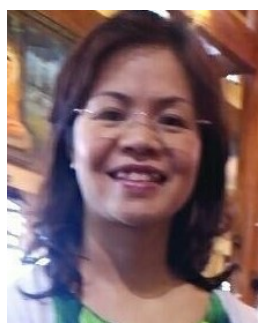

Nguyen Thi Anh-Dao was born in Thai Binh, Vietnam, in 1981. She got his Bachelor degree in Electronics - Telecommunications Technology in 2003, Master degree in Electronics Telecommunications Technology in 2013 and since November 2013, she has been a Ph.D. student in Electronics - Telecommunications Technology in 2013 from University of Engineering and Technology, Vietnam National University Hanoi.

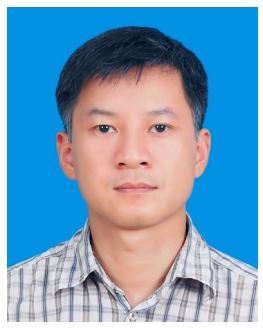

Nguyen Linh-Trung obtained his B.Eng. and Ph.D. degrees, both in Electrical Engineering, from Queensland University of Technology, Brisbane, Australia. Since 2006, he has been on the faculty of VNU University of Engineering and Technology (VNU-UET), a member university of Vietnam National University, Hanoi (VNU), where he is currently an associate professor of electronic engineering in the Faculty of Electronics and Telecommunications and director of the Advanced Institute of Engineering and Technology $(\mathrm{AVITECH})$ within VNU-UET. He is interested in signal processing methods, including time-frequency signal analysis, blind source separation, compressive sampling, tensor-based signal analysis, graph signal processing, and apply them to wireless communication and networking, biomedical engineering, with a current focus on large-scale processing.

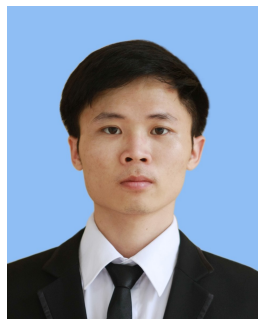

Ly Van Nguyen received the B.Eng. degree in Electronics and Telecommunications from the University of Engineering and Technology within Vietnam National University, Hano (VNU-UET), Vietnam in 2014 and the M.Sc. degree in Advanced Wireless Communications Systems from CentraleSupélec, University of Paris-Saclay, France in 2016. Since August 2017, he has been a Ph.D. student at the Computational Science Research Center (CSRC), San Diego State University (SDSU), CA, USA. His research interests include Information Theory, Wireless Communications, and Signal Processing. 


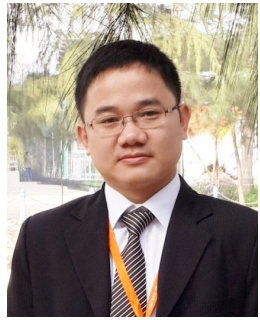

Duc-Tan Tran is an Associate professor and Vice Dean of Electronics and Telecommunication Faculty, VNU University of Engineering and Technology. He has published over 150 research papers. His publications received the "Best Paper Award" at the 9th International Conference on Multimedia and Ubiquitous Engineering (MUE-15), and the 3rd International Conference on Green and Human Information Technology (ICGHIT-2015). He was the recipient of the award for the excellent young researcher from Vietnam National University in 2008, Hanoi and the third prize in the contest "Vietnamese Talents" in 2008. His main research interests include the representation, processing, analysis, and communication of information embedded in signals and datasets. He serves as a technical committee program member, track chair, session chair and reviewer of many international conferences and journals. He is a TPC co-chair of the 2016 International Conference on Advanced Technologies for Communications.

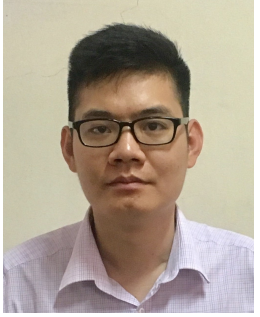

Nguyen The Hoang Anh was born in Thanh Hoa, Vietnam, in 1986. He reveived B.Sc. from National University, Hanoi, Vietnam in Electrical Engnineering in 2000 and M.S. in Electrical Engineering and M. E. in Modeling and Simulation from Old Dominion University, Norfolk, Virginia, USA in 2010 and 2012, respectively. Currently, he is a Researcher at Institute of Information Technology, Vietnam Academy of Science and Technology, Hanoi, Vietnam. His research interests include signal processing, machine learning and neuroscience.

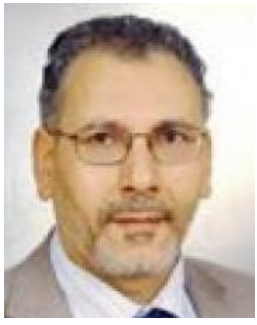

Boualem Boashash , IEEE Fellow, is a Scholar Professor, and Senior Academic with experience in five leading Universities in France and Australia. He has published more than 500 technical publications, including more than 125 journal publications covering engineering, applied mathematics, and bio-medicine. He pioneered the field of time-frequency signal processing for which he published the most comprehensive book and most powerful software package. Among many initiatives, he founded ISSPA, a leading conference since 1985. On leave from The University of Queensland, he took an appointment in the UAE as the Dean of Engineering, then moved to Qatar University as a Research Professor until 2017 when he returned to The University of Queensland. At the time of publication, his work had been cited more than 14,000 times. 